\title{
miR-217 suppresses proliferation, migration, and invasion promoting apoptosis via targeting MTDH in hepatocellular carcinoma
}

\author{
MAO ZHANG ${ }^{1,2}$, MIN LI $^{3}$, NA LI $^{4}$, ZILANG ZHANG $^{1}$, NING LIU $^{1}$, \\ XIAOYU HAN ${ }^{1}$, QINCHENG LIU $^{1}$ and CAIXIAN LIAO ${ }^{1}$
}

\author{
${ }^{1}$ Department of Hepatobiliary Surgery, Nanfang Hospital, Southern Medical University, Guangzhou, \\ Guangdong 510515; ${ }^{2}$ Department of General Surgery, Baotou Central Hospital, Baotou, Inner Mongolia 014040; \\ ${ }^{3}$ Department of General Surgery, Shilong People's Hospital, Southern Medical University, Dongguan, Guangdong 523326; \\ ${ }^{4}$ Department of Obstetrics and Gynecology, Baotou City Central Hospital, Baotou, Inner Mongolia 014040, P.R. China
}

Received August 6, 2016; Accepted December 27, 2016

DOI: $10.3892 /$ or.2017.5401

\begin{abstract}
Hepatocellular carcinoma (HCC) has frequent incidence and the third highest mortality rate among cancers in the world. This study aimed to clarify the roles of miR-217 and metadherin (MTDH) in HCC. First, we identified that miR-217 expression was downregulated and MTDH expression was upregulated in the HCC tissues. Functional studies revealed that miR-217 negatively regulated MTDH expression via binding to the 3'-untranslated region of MTDH mRNA in the HCC cells. In our further studies, the miR-217 overexpression resulted in downregulation of MTDH expression in HCC cells. The miR-217 overexpression in HCC cells suppressed proliferation, migration, and invasion inducing apoptosis. Taken together, our study provides the initial evidence that the increase of MTDH expression is associated with the decrease of miR-217 expression in HCC. This study also suggests that miR-217 inhibits malignant progression of HCC in vitro and may be used for miRNA-based therapy, possibly via directly targeting MTDH.
\end{abstract}

\section{Introduction}

As one of the most common human malignancies, hepatocellular carcinoma (HCC) possesses the third highest mortality rate (1). The therapies for HCC treatment have been notably promoted. Whereas, the overall rate of 5-year survival in HCC patients is only $12 \%$, which remains dismal (2). Surgical

Correspondence to: Dr Caixian Liao, Department of Hepatobiliary Surgery, Nanfang Hospital, Southern Medical University, 1838 North Guangzhou Avenue, Guangzhou, Guangdong 510515, P.R. China E-mail: liaocx@fimmu.com

Key words: miR-217, hepatocellular carcinoma, metadherin, proliferation, migration and invasion, apoptosis resection, radiofrequency ablation and liver transplantation may benefit some patients with early-stage HCC. However, advanced-stage HCC is diagnosed in most patients (3). Therefore, the development of new strategies for better understanding of HCC and improving efficiency of HCC therapy are anticipated.

Recently, the dysregulated microRNAs (miRNAs or miRs) in HCC have been highlighted (4). miRNAs are a family of non-coding RNAs, which comprise 20 25 nucleotides and are endogenous and conserved. Messenger RNA (mRNA) transcription can be suppressed by miRNAs, which directly target the 3'-untranslated regions (3'-UTRs) of complementary mRNAs in eukaryotes $(5,6)$. Accumulating research suggests that a diversity of biologic processes involve miRNAs, such as carcinogenesis, differentiation, apoptosis, infection, and immunity (7-9). Furthermore, it has been reported that the dysregulation of certain miRNAs are related to the progression and clinical outcomes of diverse cancers (10-12). By targeting the complementary genes, miRNAs are able to regulate cancer cell proliferation, migration, invasion and apoptosis (13), thereby suggesting that miRNAs play critical roles in cancers and probably provide a promising new way to treat cancer. In HCC, some miRNAs such as miR-145, miR133a (1), miR-144 (14) and miR-506 (15) have been reported with aberrant expression. Nevertheless, the roles of the dysregulated miRNAs and more specific miRNAs in HCC are still not well understood. miR-217 has been acknowledged as an inhibitor in various cancers including osteosarcoma $(16,17)$, lung cancer (18), pancreatic cancer (19) and clear cell renal cell carcinoma (20). Moreover, it was reported that miR-217 inhibited invasion of HCC (21). However, the detailed regulation mechanism of miR-217 in HCC is still under investigation.

Metadherin (MTDH), a 582-amino acid single pass transmembrane protein, is also known as lysine-rich CEACAM1 co-isolated (LYRIC) and astrocyte elevated gene-1 (AEG-1) (22). Since its initial cloning in 2002 (23), plenty of studies have demonstrated that MTDH expression is elevated in a great diversity of cancers (24), such as hepatocellular renal cell and gallbladder carcinomas, colorectal, gastric, prostate, 
lung, breast, ovarian, esophageal cancers and melanoma, glioma, neuroblastoma and osteosarcoma. With further studies, the expression of MTDH is associated with the development of cancers and the high expression is especially found in the aggressive metastatic stage (25). It was also identified that the overall survival rate and prognosis were poorer in HCC patients with MTDH overexpression (22). MTDH is overexpressed in $90 \%$ of HCC patients and plays a pivotal role in HCC (26), which suggests that MTDH may serve as an ideal target for anti-HCC therapy. However, the underlying molecular mechanisms of miR-217 and MTDH in HCC are poorly studied. The current research aimed to explore the roles of miR-217 and MTDH and the potential mechanisms regulating MTDH expression via miR-217 in HCC.

\section{Materials and methods}

Tissue specimen collection. HCC tissues and the normal adjacent liver tissues were collected from 20 patients in Nanfang Hospital, Southern Medical University (Guangzhou, China). All the tissues were collected before any therapy. The Ethics Committee of Nanfang Hospital, Southern Medical University approved the research. Each patient signed the informed consent.

Cell cultures. The human hepatocellular carcinoma cell line (HepG2) was purchased from the American Type Culture Collection (ATCC) (Rockville, MD, USA). Cells were cultured in Dulbecco's modified Eagle's medium (DMEM) with $10 \%$ fetal bovine serum (FBS), $100 \mu \mathrm{g} / \mathrm{ml}$ streptomycin and $100 \mathrm{U} / \mathrm{ml}$ penicillin (Gibco, Carlsbad, CA, USA). The cells were incubated at $37^{\circ} \mathrm{C}$ in a moist atmosphere containing $5 \% \mathrm{CO}_{2}$.

Quantitative real-time $R T-P C R$ ( $q R T-P C R)$. RNA extraction from the tissues or cells was by TRIzol reagent obtained from Invitrogen (Carlsbad, CA, USA). The TaqMan MicroRNA Assays (Invitrogen) were applied to quantitate the relative expression of miR-217 and the standard SYBR Green RT-PCR kit (Takara Shuzo, Kyoto, Japan) was used to detect the mRNA expression of MTDH via qRT-PCR. Primers assigned for miR-217 and MTDH were obtained from GeneCopoeia (Rockville, MD, USA). Values were normalized to either small nucleolar RNA U6 or GAPDH.

Cell transfection. GenePharma (Jiangsu, China) provided the miR-217 mimics and negative control (NC) RNA oligonucleotides. Cells were transfected with miR-217 or NC mimics (100 $\mathrm{nM}$ ) by Lipofectamine 2000 (Invitrogen) when 70-80\% cell confluence in 6-well plates. After the transfection for $36 \mathrm{~h}$, cells were collected for further tests.

Dual luciferase reporter assay. The expression plasmid for MTDH 3'-UTR wild-type or mutation and miR-217 were transfected into HepG2 cells. The firefly and renilla (the internal control) luciferase activities were examined by the dual-luciferase reporter assay system (Promega, Madison, WI, USA).

Western blot analysis. Proteins were extracted from HCC tissues or cells with RIPA lysis buffer and quantitated by the
BCA protein assay kit (Bio-Rad, Hercules, CA, USA). Proteins were subjected to $12 \%$ SDS-PAGE gels and electrophoretically transfered onto polyvinylidene difluoride membranes (Millipore, Billerica, MA, USA). Immunoblots were exposed to primary antibodies against MTDH or GAPDH (Santa Cruz Biotechnology, Santa Cruz, CA, USA) at $4^{\circ} \mathrm{C}$ overnight. Then HRP-conjugated secondary antibodies were added to analyze the immunoreactive bands with the chemiluminescence reagent (Western Lightning, Perkin Elmer Life Sciences, Boston, MA, USA).

Cell proliferation assay. Cells were seeded in a 96-well plate and cultured for $24,48,72$ or $96 \mathrm{~h}$ at $37^{\circ} \mathrm{C}$ with $5 \% \mathrm{CO}_{2}$. The cell counting kit-8 (CCK-8) (Dojindo Laboratories, Kumamoto, Japan) was used to assess the cell proliferation. The numerical values obtained on an enzyme-labeled instrument (Thermo Fisher Scientific, Bonn, German) with $450 \mathrm{~nm}$ wavelength were used to evaluate the cell viability.

Apoptosis assay. Cells were trypsinised, collected in PBS and then fixed in $70 \%$ ethanol $\left(4^{\circ} \mathrm{C}\right.$, overnight $)$, then washed with PBS and collected by centrifugation (1000 rpm, $5 \mathrm{~min})$. The Annexin V-FITC apoptosis detection kit (Beyotime, Jiangsu, China) was used to analyze apoptotic cells via flow cytometry (BD FACSCalibur).

Immunofluorescence. Sells were seeded on coverslips (Nalge Nunc International, Penfield, NY, USA) in a 24-well plate and allowed to adhere overnight. then fixed with $4 \%$ paraformaldehyde and incubated with primary anti-MTDH antibody (Invitrogen) overnight at $4^{\circ} \mathrm{C}$. Cy3-conjugated secondary antibody was added and maintained at room temperature for $1 \mathrm{~h}$. After washing the slides were counterstained with DAPI (Sigma-Aldrich). A confocal microscope (Olympus Corp., Tokyo, Japan) was used to obtain the fluorescence images.

Cell migration and invasion assays. Boyden chamber Transwells ( $8 \mu \mathrm{m}$, Millipore) was used to assess cell migration and invasion. The upper chamber with uncoated membrane (migration assay) or the membrane pre-coated with $100 \mu \mathrm{g}$ Matrigel (invasion assay) was added with $200 \mu$ l cell suspension $\left(1 \times 10^{5}\right.$ cells $\left./ \mathrm{ml}\right)$. The lower chamber was added with $600 \mu 1$ DMEM and 10\% FBS as a chemoattractant. The nonfiltered cells were gently removed and fixed with $4 \%$ paraformaldehyde after incubation for $24 \mathrm{~h}$ at $37^{\circ} \mathrm{C}$. Crystal violet $(0.1 \%)$ (Sigma-Aldrich) was applied to stain the lower chamber with filtered cells. Quantitation was performed with a microscope (Olympus Corp.).

Statistical analysis. The data are presented as mean \pm SD. Student's t-test was used to evaluate differences between the stimulated sample and the respective control. For multiple comparisons, statistically significant differences were assessed via one-way ANOVA. P-value $<0.05$ was deemed statistically significant.

\section{Results}

miR-217 expression decreases and MTDH expression increases in HCC tissues. As depicted in Fig. 1A, compared 
A

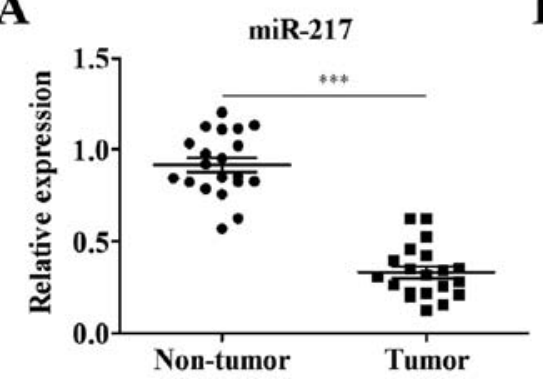

B

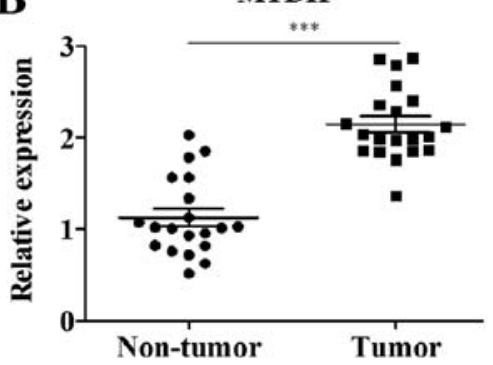

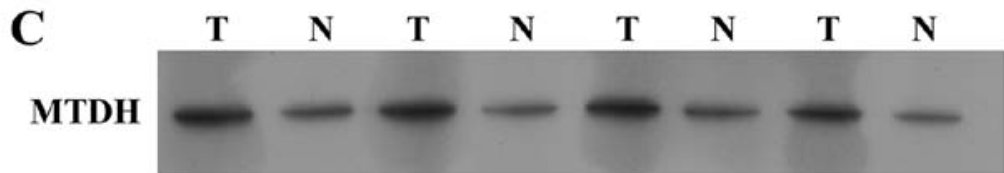

GAPDH

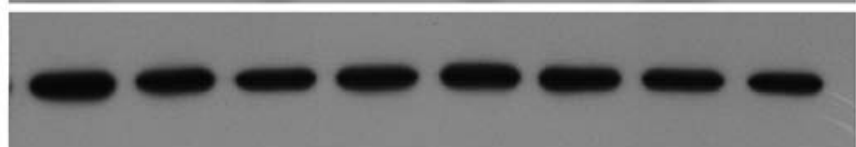

Figure 1. Expression of miR-217 and MTDH in HCC tissues. (A) The mRNA expression levels of miR-217 in 20 pairs of HCC tissues (tumor) and the matched adjacent normal tissues (non-tumor) were detected by quantitative RT-PCR. miR-217 expression was significantly lower in HCC tissues than in the corresponding noncancerous liver tissues. (B) The mRNA expression of MTDH was notably upregulated in HCC tissues compared with the normal tissues. (C) The protein expression of MTDH was detected in HCC tissues (T) and the matched normal tissues (N) by western blotting, the matched N/T cases had increased levels of MTDH in the HCC sample compared with the adjacent normal tissues. ${ }^{* * * *} \mathrm{P}<0.0001$.

A

\section{MTDH WT 3'UTR}

$\operatorname{miR}-217$

B

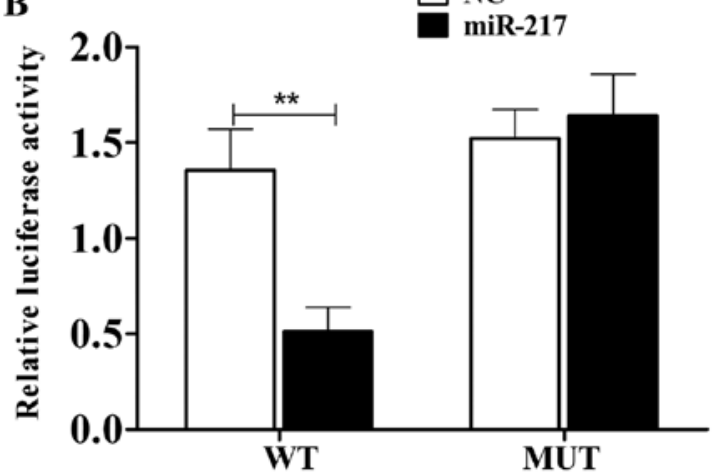

MTDH MUT 3'UTR

Figure 2. miR-217 directly targeted MTDH in HCC cells. (A) The putative binding sites of miR-217 within the 3'-UTR of MTDH wt (wild-type) are indicated. (B) Relative luciferase activity of the indicated MTDH reporter construct. The luciferase activity was suppressed in the cells cotransfected with MTDH-wt and miR-217. The results are presented as the mean \pm SD of three independent experiments; ${ }^{* *} \mathrm{P}<0.01$ versus the group (miR-217-, MTDH-wt+).

with the matched normal tissues, miR-217 expression level in HCC tissues significantly decreased $(\mathrm{P}<0.0001)$. Moreover, the expression of MTDH was examined via qRT-PCR and western blotting in HCC tissues. Compared with the normal tissues, the mRNA expression of MTDH was significantly upregulated in HCC tissues (Fig. 1B), as well as the protein expression of MTDH (Fig. 1C).
miR-217 targets MTDH in HCC cells. As miR-217 and MTDH expressed negatively in HCC, we investigated whether miR-217 can target MTDH. As shown in Fig. 2A, miR-217 bound to the 3'-UTR of MTDH gene. The dual luciferase reporter assay was further performed to confirm their relationship. As displayed in Fig. 2B, after cotransfection with the 3'-UTR of wild-type MTDH and miR-217, the relative luciferase activity was remarkably decreased in HepG 2 cells $(\mathrm{P}<0.05)$. While the luciferase activity of the mutant construct showed little change, indicating that miR-217 inhibited MTDH transcription via directly targeting the 3'-UTR of MTDH.

miR-217 negatively regulates MTDH expression in HCC cells. To further validate the prediction of the suppression effect of miR-217 on MTDH gene, we explored whether miR-217 could regulate the expression of endogenous MTDH in HepG2 cells. As depicted in Fig. 3A and B, compared with the control or mimics NC, the miR-217 expression in the cells transfected with miR-217 mimics was notablely upregulated $(\mathrm{P}<0.01)$, indicating that the transfection efficiency was satisfactory. We next examined the MTDH mRNA expression and observed a remarkably downregulation after transfection with miR-217 mimics $(\mathrm{P}<0.01)$. Furthermore, we found by western blotting that the MTDH expression in the HepG2 cells with miR-217 overexpressed was markedly reduced compared with the control or mimics NC (Fig. 3C) . Similar results were obtained by immunofluorescence assays (Fig. 4).

miR-217 suppresses proliferation and promotes apoptosis in HCC cells. miR-217 or NC mimics were transfected into HepG2 cells to explore the role of miR-217 in HCC cells. As shown in Fig. 5A, the proliferation of miR-217-overexpressed cells was inhibited compared with the control or mimics NC. Moreover, the cell cycle showed similar results (Fig. 5B). These 

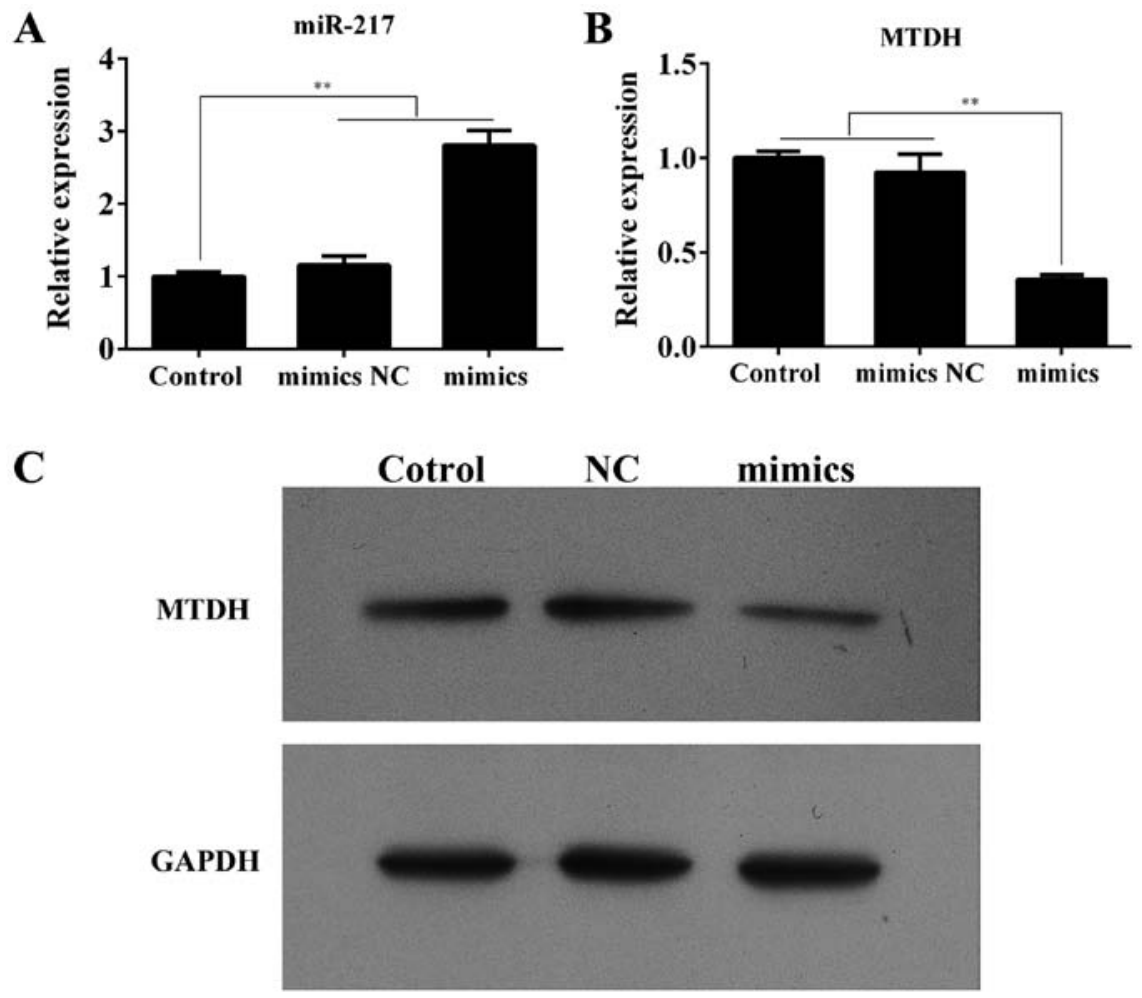

Figure 3. miR-217 negatively regulates MTDH expression in HCC cells. (A) Quantitative RT-PCR was performed to detect the mRNA expression of miR-217 upon transfection with miR-217 mimics. (B) The mRNA expression of MTDH in HepG2 cells transfected with miR-217 mimics. (C) The MTDH protein expression levels were detected by western blotting in HepG2 cells transfected with mimics. Each value represents the mean $\pm \mathrm{SD} ; \mathrm{n}=3$. ${ }^{* *} \mathrm{P}<0.01$.

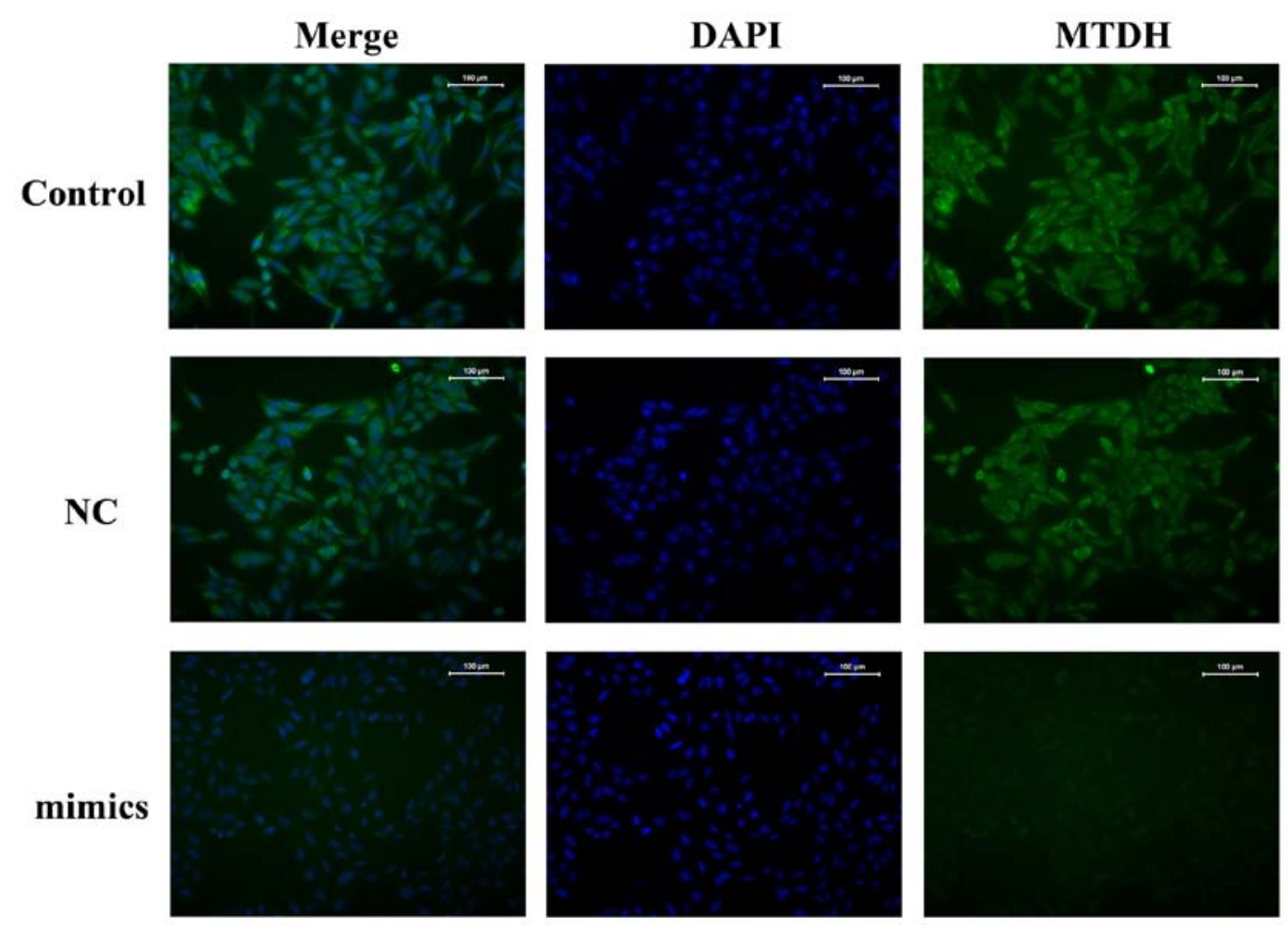

Figure 4. Expression and localization of MTDH in HCC cells by immunofluorescence staining. Green fluorescence represents the expression of MTDH. Blue fluorescence represents DAPI staining in karyotin. Magnification, x100; scale bar, $100 \mu \mathrm{m}$.

data suggest that miR-217 played a proliferation suppressing role in HCC. In addition, we explored the effect of miR-217 upregulation on apoptosis in HCC. Compared with the control groups, the cell apoptosis notably increased in miR-217-upregulated HepG2 cells (Fig. 5C and D), indicating that miR-217 expression promoted apoptosis of HCC cells. 
A

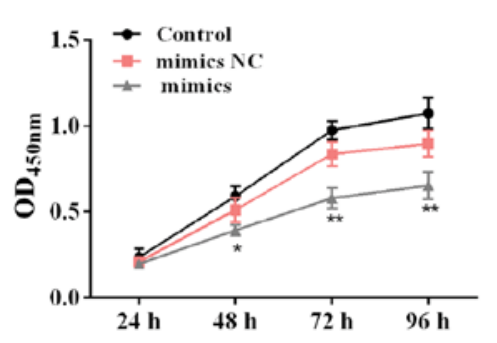

D

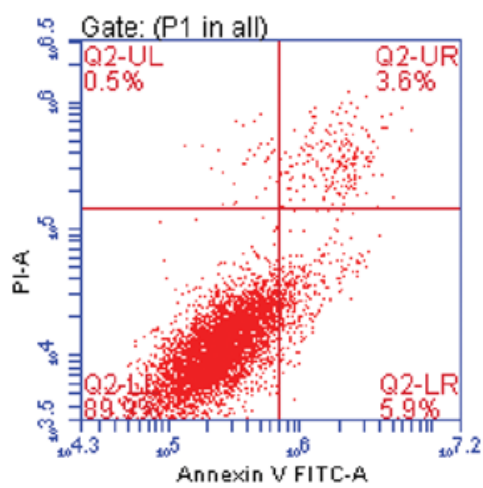

B

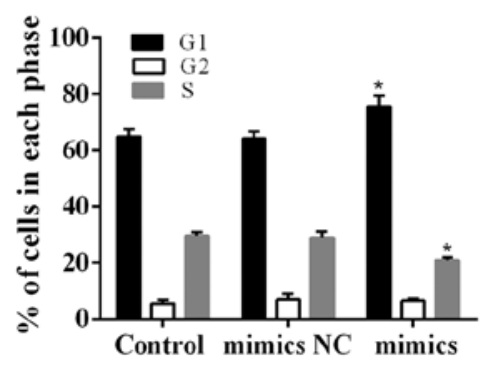

mimes NC

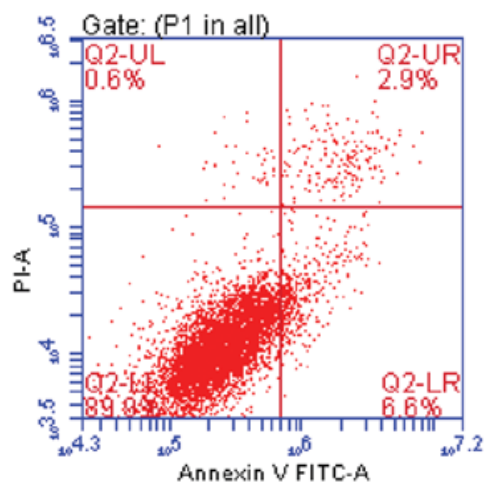

C

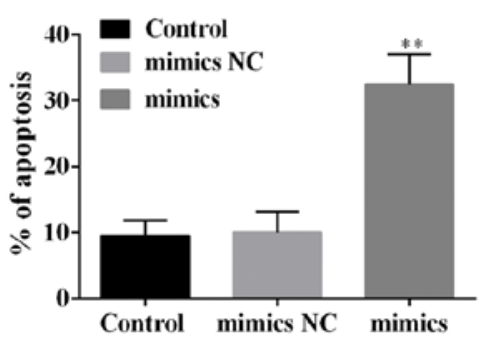

mimics

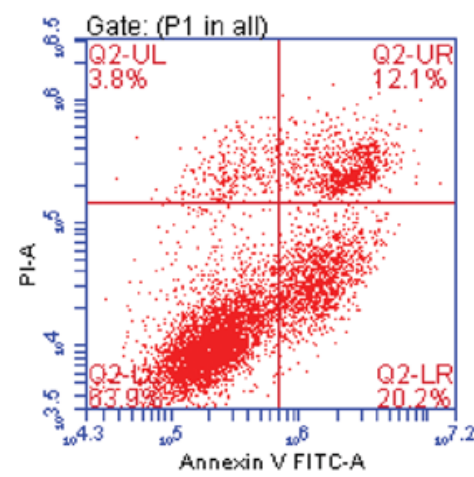

Figure 5. miR-217 inhibits HCC cell proliferation and induces cell apoptosis. (A) Cell proliferation was measured via CCK-8 assay after transfection in HepG2 cells. (B) Cell cycle was evaluated by flow cytometer in HepG2 cells. (C) Cell apoptosis was evaluated by flow cytometer in HepG2 cells. (D) Representative graphs of the apoptosis assay. Each point represents the mean $\pm \mathrm{SD} ; \mathrm{n}=3 .{ }^{*} \mathrm{P}<0.05 ;{ }^{* *} \mathrm{P}<0.01$.

A

Control

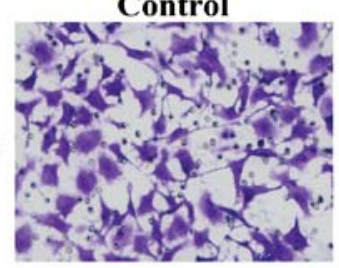

Migration

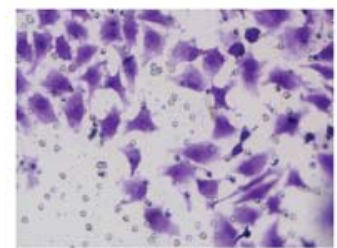

B

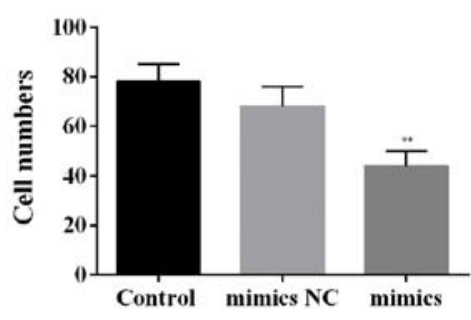

Migration mimes NC
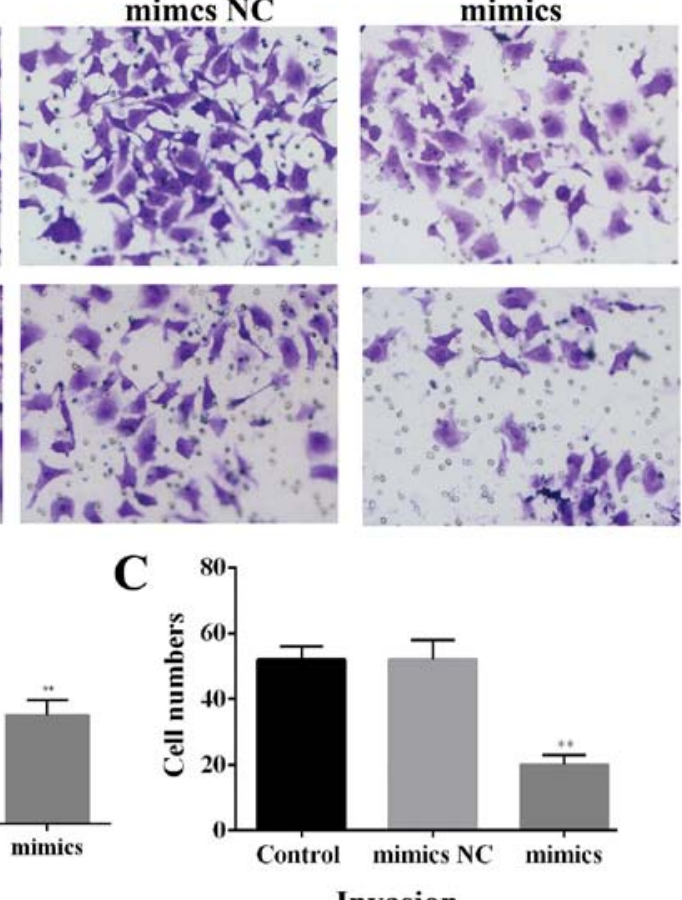

Invasion

Figure 6. Effects of miR-217 on HCC cell migration and invasion. Cell migration and invasion were examined by Transwell assay in HepG2 cells. (A) The representative images in each group were evaluated by microscopy. Magnification, $x 400$. (B) The migrated cells in each field were counted and summarized. (C) The invasive cells in each field were counted and summarized. The results are expressed as the mean $\pm \mathrm{SD} ; \mathrm{n}=3 ;{ }^{* *} \mathrm{P}<0.01$.

miR-217 restraines migration and invasion in $H C C$ cells. As displayed in Fig. 6A, the HepG2 cells overexpressing miR-217 had lower migration and invasion compared with the control groups. Moreover, the statistical results demonstrated that 
miR-217 overexpression markedly suppressed migration and invasion in HCC cells (Fig. 6B and C).

\section{Discussion}

$\mathrm{HCC}$ is well known as one of the most lethal cancers globally for its low cure rate, high recurrence rate and high mortality (15). The development of miRNAs provides a potential novel choice for HCC diagnosis and therapy (27). miR-217 expression has been reported to be lower in cancer cells, however the role of miR-217 in HCC has not been well investigated (28). To further identify the potential function and molecular mechanism of miR-217 in HCC, we used human HCC tissues or normal liver tissues to test miR-217 expression. As expected, the miR-217 expression in HCC tissues was remarkably decreased. These data suggest that miR-217 possibly serves as a new marker for HCC diagnosis or a novel target for HCC therapy.

Accumulating convincing studies have pointed out that MTDH may act as a pivotal element to cancer onset and progression (29). It has been reported that MTDH is markedly upregulated in HCC patients and could be a novel serum biomarker for HCC (30). MTDH has been demonstrated to be a potential critical gene regulating various biochemical phenotypes of cancer progression, such as metastasis, chemoresistance, transformation and evasion of apoptosis (31). This study concurs that the expression levels of both mRNA and protein MTDH were significantly upregulated in the $20 \mathrm{HCC}$ tissues compared to the matched normal adjacent tissues. MTDH contributes to HCC initiation and progression by a variety of mechanisms, such as interference with thyroid hormone (T3) function, downregulation of type I 5'-deiodinase (DIO1) level, a local hypothyroid state creation in the liver, activation of nuclear factor $\kappa \mathrm{B}(\mathrm{NF}-\kappa \mathrm{B})$ and other protumorigenic signaling pathways (32). Knockdown of MTDH inhibits proliferation, invasion and notably abolishes cancer onset, growth and metastasis $(33,34)$. Thus, MTDH inhibition might be an effective way to counteract this fatal malady for which there is no effective treatment. MTDH inhibition will not only eliminate HCC but also ameliorate detrimental effects associated with the disease such as non-thyroidal illness syndrome (NTIS). Efforts need to be spent on the development of MTDH inhibitors in the clinic.

miRNAs perform as an essential component in cancer initiation and development via targeting and then decreasing the expression of key regulator (15). The present investigation was carried out to determine the relationship between miR-217 and MTDH. The findings show that miR-217 directly targeted 3'-UTR of MTDH, inhibiting both the mRNA and protein expression levels of MTDH. Moreover, miR-217 acts as an MTDH inhibitor in HCC. Thus, the cellular function of miR-217 was further examined. The miR-217 overexpression notablely restrained proliferation and promoted apoptosis of HCC cells. Furthermore, migration and invasion strongly decreased in miR-217-transfected cells. Taken together, this study indicates that miR-217 inhibits proliferation, migration, invasion inducing apoptosis possibly by targeting MTDH in HCC. The current study also revealed that miR-217 shows little effect on the cell cycle, suggesting the tumor suppression effect of miR-217 is not associated with cell cycle in
HCC. As each miRNA may regulate several genes and each gene possibly is regulated by a variety of miRNAs, the detailed mechanism and upstream regulators of MTDH in HCC still need more exploration. In addition, we must point out that this study was executed in a single cell line and thus, more confirmation is needed.

In conclusion, this study indicates that miR-217 downregulates the expression of $\mathrm{MTDH}$, a key regulator of tumor proliferation, migration and invasion in HCC cells. The findings in this study also encourage us to develop miR-217 as a new potential target for diagnosis and gene therapy of HCC.

\section{References}

1. Wang G, Zhu S, Gu Y, Chen Q, Liu X and Fu H: MicroRNA-145 and microRNA-133a inhibited proliferation, migration, and invasion, while promoted apoptosis in hepatocellular carcinoma cells via targeting FSCN1. Dig Dis Sci 60: 3044-3052, 2015.

2. Deng Q, Xie L and Li H: MiR-506 suppresses cell proliferation and tumor growth by targeting Rho-associated protein kinase 1 in hepatocellular carcinoma. Biochem Biophys Res Commun 467: 921-927, 2015.

3. Singal AG, Nehra M, Adams-Huet B, Yopp AC, Tiro JA, Marrero JA, Lok AS and Lee WM: Detection of hepatocellular carcinoma at advanced stages among patients in the HALT-C trial: Where did surveillance fail? Am J Gastroenterol 108: 425-432, 2013.

4. Callegari E, Gramantieri L, Domenicali M, D'Abundo L, Sabbioni S and Negrini M: MicroRNAs in liver cancer: A model for investigating pathogenesis and novel therapeutic approaches. Cell Death Differ 22: 46-57, 2015.

5. Bartel DP: MicroRNAs: Target recognition and regulatory functions. Cell 136: 215-233, 2009.

6. Inui M, Martello G and Piccolo S: MicroRNA control of signal transduction. Nat Rev Mol Cell Biol 11: 252-263, 2010.

7. Liu AM, Xu Z, Shek FH, Wong KF, Lee NP, Poon RT, Chen J and Luk JM: miR-122 targets pyruvate kinase M2 and affects metabolism of hepatocellular carcinoma. PLoS One 9: e86872, 2014.

8. Yang N, Ekanem NR, Sakyi CA and Ray SD: Hepatocellular carcinoma and microRNA: New perspectives on therapeutics and diagnostics. Adv Drug Deliv Rev 81: 62-74, 2015.

9. Bartel DP: MicroRNAs: Genomics, biogenesis, mechanism, and function. Cell 116: 281-297, 2004.

10. Ell B and Kang Y: MicroRNAs as regulators of bone homeostasis and bone metastasis. Bonekey Rep 3: 549, 2014.

11. Khan K, Cunningham D, Peckitt C, Barton S, Tait D, Hawkins M, Watkins D, Starling N, Rao S, Begum R, et al: miR-21 expression and clinical outcome in locally advanced pancreatic cancer: Exploratory analysis of the pancreatic cancer Erbitux, radiotherapy and UFT (PERU) trial. Oncotarget 7: 12672-12681, 2016.

12. van Rooij E and Kauppinen S: Development of microRNA therapeutics is coming of age. EMBO Mol Med 6: 851-864, 2014.

13. Borel F, Konstantinova P and Jansen PL: Diagnostic and therapeutic potential of miRNA signatures in patients with hepatocellular carcinoma. J Hepatol 56: 1371-1383, 2012.

14. Cao T, Li H, Hu Y, Ma D and Cai X: miR-144 suppresses the proliferation and metastasis of hepatocellular carcinoma by targeting E2F3. Tumour Biol 35: 10759-10764, 2014.

15. Dai W, Huang HL, Hu M, Wang SJ, He HJ, Chen NP and Li MY: microRNA-506 regulates proliferation, migration and invasion in hepatocellular carcinoma by targeting F-spondin 1 (SPON1). Am J Cancer Res 5: 2697-2707, 2015.

16. Sun B, Yang M, Li M and Wang F: The microRNA-217 functions as a tumor suppressor and is frequently downregulated in human osteosarcoma. Biomed Pharmacother 71: 58-63, 2015.

17. Shen L, Wang P, Yang J and Li X: MicroRNA-217 regulates WASF3 expression and suppresses tumor growth and metastasis in osteosarcoma. PLoS One 9: e109138, 2014.

18. Guo J, Feng Z, Huang Z, Wang H and Lu W: MicroRNA-217 functions as a tumour suppressor gene and correlates with cell resistance to cisplatin in lung cancer. Mol Cells 37: 664-671, 2014. 
19. Zhao WG, Yu SN, Lu ZH, Ma YH, Gu YM and Chen J: The miR-217 microRNA functions as a potential tumor suppressor in pancreatic ductal adenocarcinoma by targeting KRAS. Carcinogenesis 31: 1726-1733, 2010.

20. Li H, Zhao J, Zhang JW, Huang QY, Huang JZ, Chi LS, Tang HJ, Liu GQ, Zhu DJ and Ma WM: MicroRNA-217, down-regulated in clear cell renal cell carcinoma and associated with lower survival, suppresses cell proliferation and migration. Neoplasma 60: 511-515, 2013.

21. Su J, Wang Q, Liu Y and Zhong M: miR-217 inhibits invasion of hepatocellular carcinoma cells through direct suppression of E2F3. Mol Cell Biochem 392: 289-296, 2014.

22. Li WF, Ou Q, Dai H and Liu CA: Lentiviral-mediated short hairpin RNA knockdown of MTDH inhibits cell growth and induces apoptosis by regulating the PTEN/AKT pathway in hepatocellular carcinoma. Int J Mol Sci 16: 19419-19432, 2015.

23. Su ZZ, Kang DC, Chen Y, Pekarskaya O, Chao W, Volsky DJ and Fisher PB: Identification and cloning of human astrocyte genes displaying elevated expression after infection with HIV-1 or exposure to HIV-1 envelope glycoprotein by rapid subtraction hybridization, RaSH. Oncogene 21: 3592-3602, 2002.

24. Shi $X$ and Wang X: The role of MTDH/AEG-1 in the progression of cancer. Int J Clin Exp Med 8: 4795-4807, 2015.

25. Sarkar D and Fisher PB: AEG-1/MTDH/LYRIC: Clinical significance. Adv Cancer Res 120: 39-74, 2013.

26. Yoo BK, Emdad L, Su ZZ, Villanueva A, Chiang DY, Mukhopadhyay ND, Mills AS, Waxman S, Fisher RA, Llovet JM, et al: Astrocyte elevated gene-1 regulates hepatocellular carcinoma development and progression. J Clin Invest 119: 465-477, 2009.

27. Wei R, Deng Z and Su J: miR-217 targeting Wnt5a in osteosarcoma functions as a potential tumor suppressor. Biomed Pharmacother 72: 158-164, 2015.
28. Wang B, Shen ZL, Jiang KW, Zhao G, Wang CY, Yan YC, Yang Y, Zhang JZ, Shen C, Gao ZD, et al: MicroRNA-217 functions as a prognosis predictor and inhibits colorectal cancer cell proliferation and invasion via an AEG-1 dependent mechanism. BMC Cancer 15: 437, 2015.

29. Robertson CL, Srivastava J, Rajasekaran D, Gredler R, Akiel MA, Jariwala N, Siddiq A, Emdad L, Fisher PB and Sarkar D: The role of AEG-1 in the development of liver cancer. Hepat Oncol 2: 303-312, 2015

30. Wang G, Yang J, Cao S, Li J and Liu B: AEG-1 acts as a novel bio-marker in the diagnosis of patients with hepatocellular carcinoma. Int J Clin Exp Pathol 9: 1940-1946, 2016.

31. Zhou Z, Deng H, Yan W, Huang H, Deng Y, Li Y and Tian D: Expression of metadherin/AEG-1 gene is positively related to orientation chemotaxis and adhesion of human hepatocellular carcinoma cell lines of different metastatic potentials. J Huazhong Univ Sci Technolog Med Sci 32: 353-357, 2012.

32. Srivastava J, Robertson CL, Gredler R, Siddiq A, Rajasekaran D, Akiel MA, Emdad L, Mas V, Mukhopadhyay ND, Fisher PB, et al: Astrocyte elevated gene-1 (AEG-1) contributes to nonthyroidal illness syndrome (NTIS) associated with hepatocellular carcinoma (HCC). J Biol Chem 290: 15549-15558, 2015.

33. Robertson CL, Srivastava J, Siddiq A, Gredler R, Emdad L, Rajasekaran D, Akiel M, Shen XN, Guo C, Giashuddin S, et al: Genetic deletion of AEG-1 prevents hepatocarcinogenesis. Cancer Res 74: 6184-6193, 2014.

34. Wan L, Hu G, Wei Y, Yuan M, Bronson RT, Yang Q, Siddiqui J, Pienta KJ and Kang Y: Genetic ablation of metadherin inhibits autochthonous prostate cancer progression and metastasis. Cancer Res 74: 5336-5347, 2014. 